\title{
Article \\ Coordinated Speed Control Strategy for Minimizing Energy Consumption of a Shearer in Fully Mechanized Mining ${ }^{\dagger}$
}

\author{
Zheng Zheng ${ }^{1}\left(\mathbb{D}\right.$, Dilei Chen ${ }^{1, * \mathbb{D}}$, Tao Huang ${ }^{2, * \mathbb{D}}$ and Guopeng Zhang ${ }^{1}$ \\ 1 School of Electrical Engineering and Automation, Henan Polytechnic University, Jiaozuo 454003, China; \\ zhengzh@hpu.edu.cn (Z.Z.); zhangguopeng@hpu.edu.cn (G.Z.) \\ 2 Department of Energy, Politecnico di Torino, 10129 Turin, Italy \\ * Correspondence: 111907010003@home.hpu.edu.cn (D.C.); tao.huang@polito.it (T.H.); \\ Tel.: +39-011-090-7117 (D.C. \& T.H.) \\ + This paper is an extended version of our paper published in 2020 55th International Universities Power \\ En-gineering Conference (UPEC), Turin, Italy, 2020, pp. 1-6.
}

Citation: Zheng, Z.; Chen, D.; Huang, T.; Zhang, G. Coordinated Speed Control Strategy for Minimizing Energy Consumption of a Shearer in Fully Mechanized Mining . Energies 2021, 14, 1224. https://doi.org/10.3390/en14051224

Academic Editor:

Dimitrios Katsaprakakis

Received: 30 January 2021

Accepted: 19 February 2021

Published: 24 February 2021

Publisher's Note: MDPI stays neutral with regard to jurisdictional claims in published maps and institutional affiliations.

Copyright: (C) 2021 by the authors Licensee MDPI, Basel, Switzerland. This article is an open access article distributed under the terms and conditions of the Creative Commons Attribution (CC BY) license (https:/ / creativecommons.org/licenses/by/ $4.0 /)$.

\begin{abstract}
As one of the major pieces of equipment in fully mechanized coal mining, the drum shearer plays a critical role in improving the efficiency and energy utilization in the coal mining production process. In this paper, an energy consumption model of a shearer, derived from the analysis of the cutting and traction resistances on the shearer during different processes within a working cycle, is established. Based on the derived model, control and coordination strategies between the two speeds are proposed to minimize the shearer's energy consumption in unidirectional mining. The case study of a real coal mine shows that the proposed models are valid, and the optimal control of shearer speeds can effectively reduce the energy consumption by $5.16 \%$ in a working cycle. To gain further insights into the impact of traction speed and drum rotational speed on the shearer's energy consumption, several speed coordination cases are employed to further compare with the optimized one. Our study results show that the energy consumption of a shearer can be decreased with the increase of traction speed while decreasing drum rotational speed in coordination.
\end{abstract}

Keywords: shearer; coal mining; energy consumption; traction speed; drum rotational speed; optimal control

\section{Introduction}

It is widely recognized that energy security is critical for the development and prosperity of a nation and the world. Even though the energy transition has fast developed in many countries, among traditional fossil fuels, coal is still indispensable in many countries. For example, coal accounted for about $58 \%$ of the total energy consumption in 2020, according to China's "13th Five-Year Plan for the Development of Coal Industry" [1]. The underground environment for fully mechanized coal mining is constantly changing and the mining processes are sophisticated. The energy consumption of the mining system accounts for about $25 \%$ of the total energy consumption for coal production, which is the largest among six subsystems, namely the mining system, transportation system, lifting system, pressurized air system, drainage system and ventilation system [2]. Furthermore, the shearer, the most widely used piece of equipment in coal production, accounts for a larger proportion of energy consumption, determining the efficiency and energy consumption of coal mining directly. Therefore, it is essential to reduce the energy consumption of the shearer in coal mining, especially with the trend of energy conservation and emission of the coal industry.

Many factors can affect the energy consumption of a shearer, such as the characteristics of the coal seam, structural parameters of the shearer, coordination of two speeds and mining conditions. At present, researchers have studied the influence of different factors on a shearer, such as the relationship between coal mining time and profit, the optimization 
of longwall length, the force situation in the mining process, the optimization of operating parameters in drum cutting. The authors of reference [3] derived a mathematical model of the relationship between daily coal mining output and effective mining time in the longwall face. The authors of reference [4] used the specialized simulating model library of technological mining equipment to develop an integrated model for technological processes of underground coal mining in stoping face, and evaluated the productivity of a shearer depending on different factors such as the technical parameters of the shearer, length of the longwall face, geophysical state of the coal seam. The authors of reference [5] proposed an analytical solution to optimize the length of a longwall face aiming at maximizing the annual profit. Reference [6] focuses on longwall systems designed for seam mining, the most important assumptions and advantages of longwall exploitation are presented. In reference [7], a multi-objective optimization model of shearer was built which takes movement parameters as its design variables, then the integrated optimal movement parameters under different coal seam hardness were obtained. Reference [8] described method of assessing the quality of conical picks, and adopted a three-stage process of testing; the method should be used for evaluating the quality of picks and their selection. However, references [3-8] all failed to discuss the relationship among energy consumption, daily production, and mining time. In reference [9], the cutting performance indexes of shearer's spiral drum under various working conditions were obtained and the effects of the shearer's traction speed, drum speed, blade spiral angle and depth exerted on the cutting performance were explored. In reference [10], the energy consumption characteristics of drum-shearer were discussed and the reasonable traction speed was determined. The authors of reference [11] focused on the situation of automation and robotization of underground mining in Poland, their work including supporting the self-propelled drilling carts and roadheaders, autonomous equipment for cutting rocks, automatic longwall systems, and rescue operations. The authors of reference [12] established the cutting energy consumption model of the shearer, analyzed the factors affecting cutting energy consumption, and calculated the cutting energy consumption of shearer under different working conditions. However, the above works of [9-12] all failed to analyze cooperative speed regulation between the traction speed and the drum rotational speed, likewise the relationship with energy consumption of shearer.

In summary, there is no in depth research on the energy consumption modeling and optimal control of the mechanized mining equipment presently. Therefore, in this paper, we firstly establish an energy consumption model of a shearer in unidirectional mining as a function of the traction speed, the drum rotational speed and the effective coal mining time. This model is then transformed into an optimization model aiming at minimizing the overall energy consumption of the shearer under the requirements of the same coal mining output, and a certain period of time.

The main contributions of this paper are as follows:

(1) We propose an energy consumption model of the shearer in unidirectional mining based on the force and resistance analysis through its entire mining process. The model has a special focus on the relationship among the energy consumption and output of mining, traction speed and drum rotating speed, as well as the effective coal mining time.

(2) On the basis of the proposed model, we establish an optimal control strategy for the drum rotational speed and traction speed, with the purpose of the minimal energy consumption of the shearer in one production cycle, constrained by the working process, working load and technical limits.

(3) Detailed analysis of the impact of the drum rotational speed and traction speed on the final energy consumption is provided to give practical implications on how to possibly approach more energy-saving production if optimal speed coordination cannot be achieved during the real complex production.

The remainder of this paper is organized as follows. Section 2 describes the coal mining process of unidirectional mining. Then, the energy consumption model of a shearer 
is constructed in Section 3. Section 4 establishes the optimization problem of minimal energy consumption based on the model of Section 3. Simulations are carried out in Section 5 with comparisons among different cases for the best and practical implication of the speed coordination, Section 6 draws some conclusions.

\section{Coal-Mining Process}

The mining methods of a shearer in a fully mechanized coal-mining face can be categorized as unidirectional mining and bidirectional mining. Furthermore, concerning the shearer's feed, there can be oblique cutting at the end or in the middle of the mining face. In this paper, for simplicity and clearance for the reader, we take the most straightforward unidirectional mining scheme with oblique cutting feed at the end of the mining face as an example. However, the following discussion of technology is suitable for the unidirectional with another feeding mode or bi-directional mining with one of the two feeding modes.

A process in coal mining refers to a set of specific procedures involved in the mining task; only after finishing all the processes in a particular sequence can a complete production cycle of mining be finished. The double-drum shearer's executed process in the unidirectional coal mining in $k$-th production cycle of $m$-th fully mechanized coal-mining face is shown in Figure 1.

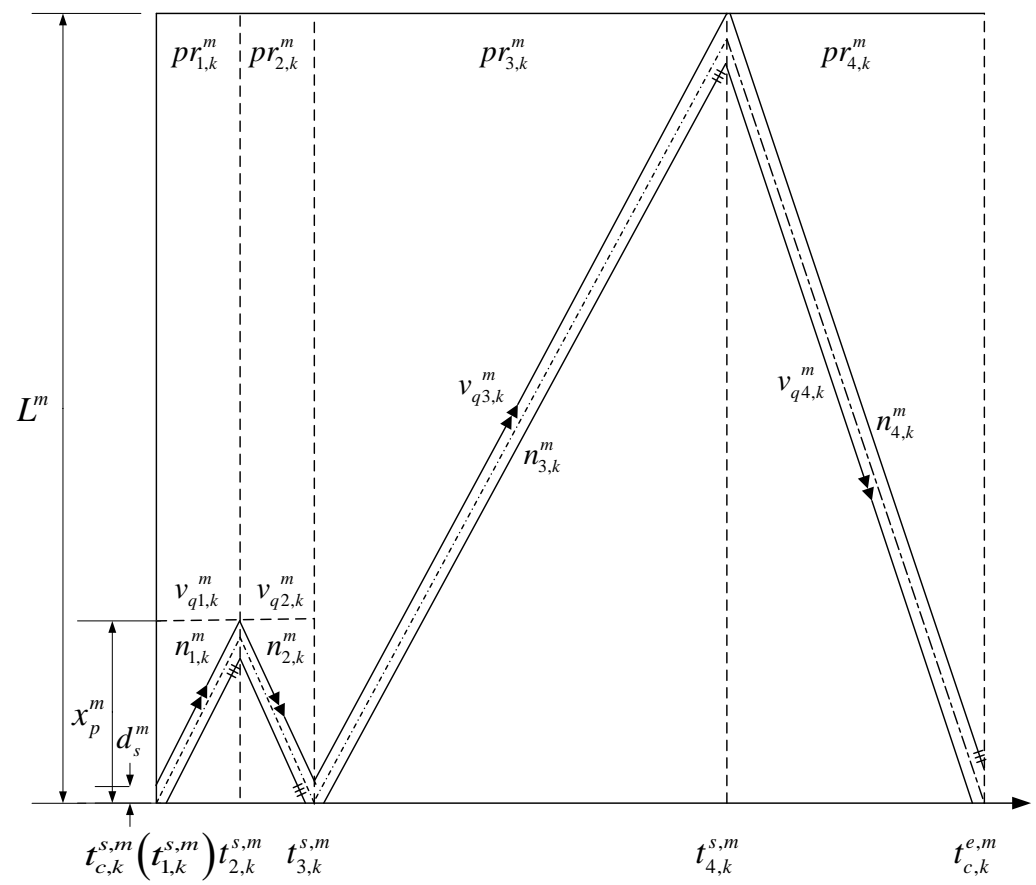

Figure 1. Working processes of a shearer in $k$-th complete cycle of unidirectional mining, adopted from [13].

The $x$-axis of Figure 1 represents the $k$-th mining cycle time, with a particular focus on the starting and ending time of each process. The $y$-axis denotes the shearer's position on the longwall. The symbol $\rightarrow$ depicts the operation of the shearer; symbol $-\cdots$ represents the moving of hydraulic support; symbol $\ldots$ stands for pushing scraper conveyor. $t_{c, k}^{s, m}$ and $t_{c, k}^{e, m}$ represent the starting and ending time of the shearer operation in $k$-th complete cycle of $m$-th fully mechanized coal mining face, respectively. $p r_{i, k}^{m}$ indicates the $i$-th process in $k$-th complete cycle of $m$-th fully mechanized coal mining face (Hereinafter, we use superscript $m$ to denote the $m$-th fully mechanized coal mining face, subscript $k$ to denote $k$-th production cycle, and subscript $i$ to denote the $i$-th process.), $t_{i, k}^{s, m}$ and $t_{i, k}^{e, m}$ represent the starting and ending time in $p_{i, k}^{m}$ the corresponding traction speed is $v_{q i, k}^{m, k}$ and drum 
rotational speed is $n_{i, k}^{m}$. Therefore, with Figure 1 , we can clearly understand the shearer's movements and positions along time when the processes proceed.

In addition, Figure 1 includes the following elements:

$L^{m}$ : longwall length [m].

$x_{p}^{m}$ : distance between shearer stoppage place and longwall roadway crossing [m].

$d_{s}^{m}$ : shearer length [m].

In order to describe each process in a unified and explicit way, we divide the processes shown in Figure 1 into three groups in the following subsections.

\subsection{Oblique Cutting and Feeding at the End of the Longwall}

$p r_{1, k}^{m}$ : The shearer oblique cutting and feeding at one end of mining face in the first process. With the traction speed $v_{q 1, k}^{m}$ drum rotational speed $n_{1, k}^{m}$, the effective operating time starts from $t_{1, k}^{s, m}$ and ends with $t_{1, k}^{e, m}$, and the shearer stops at $x_{p}^{m}$ on the longwall at the end of $\mathrm{pr}_{1, k}^{\mathrm{m}}$.

$\mathrm{pr}_{2, k}^{m}$ : The front and rear drums of shearer are changed the top and bottom positions and return to the end of mining face. With the traction speed $v_{q 2, k}^{m}$ drum rotational speed $n_{2, k}^{m}$, the effective operating time starts from $t_{2, k}^{s, m}$ and ends with $t_{2, k}^{e, m}$, and the running distance of the shearer is $x_{p}^{m}$ in $p r_{2, k}^{m}$.

\subsection{Linear Cutting from Tail to Head}

$\mathrm{pr}_{3, k}^{m}$ : The shearer turns track with empty cutting at the triangle of the mining face firstly and then starts to cut coal linearly with $v_{q 3, k}^{m}$ and $n_{3, k}^{m}$, and the shearer stops at another end of the longwall at the end of $\mathrm{pr}_{3, k}^{m}$.

\subsection{Empty Cutting}

$\mathrm{pr}_{4, k}^{m}$ : The shearer returns with an empty cutting from the other end, only the traction mechanism is in operation and the drum does not cut coal in this process. After moving the hydraulic support and pushing the scraper conveyor straight, the shearer begins the $(k+1)$-th mining cycle at the end of $p r_{4, k}^{m}$.

\section{Energy Consumption Analysis of Coal-Mining Processes}

The shearer's power source in a mining system comes from the cutting motor and traction motor. The cutting motor provides power for the drum responsible for cutting and charging the coal, and the traction motor provides power for the traction part, which supports the whole cutting mechanism and makes it move smoothly along the scraper conveyor. The energy consumption of a shearer during coal mining mainly includes the total energy consumption of drum cutting, coal charging, and traction moving. In this section, we first analyze the cutting resistance, traction resistance, cutting power and traction power, and then establish the energy consumption model of a shearer in $p r_{i, k}^{m}$.

\subsection{Force Analysis of Shearer}

\subsubsection{Instantaneous Total Average Cutting Resistance of the Drum $f_{j i, k}^{m}$}

The drum rotationally drives the conical picks, installed on the drum at different installation angles, to cut the coal. The drum's real-time load is related to various parameters, including the coal seams, structure of the picks, cutting technique, and kinematic parameters. The drum force is generally calculated by the mechanical theory and linear cutting models proposed by Be $\pi$ oh, Evans, and Nishimatsu [14] in the 1950s.

At any time, only the part of picks in contact with the coal will cut, and the cutting thickness is determined by various factors, such as traction speed, drum rotational speed, and cutting radius. Theoretically, the instantaneous cutting thickness of the drum changes according to the rule of crescent shape, and the frequency and amplitude of traction speed continuously vary according to the dynamics of the shearer and movement of the traction mechanism. In reality, the miners will adjust the traction speed according to the coal seam's 
conditions. Therefore, it is impossible and unnecessary to calculate the instantaneous cutting thickness precisely. In our analysis, we use the average cutting thickness $h_{\phi \varphi, k}^{\bar{m}}$ as Formula (1) [10]:

$$
h_{\phi \varphi, k}^{\bar{m}}=\frac{200 D_{c}^{m} v_{q i, k}^{m}}{\frac{\pi D_{c}^{m} n_{i, k}^{m}}{60} a_{\phi}^{m}}=\frac{12000 v_{q i, k}^{m}}{\pi a_{\phi}^{m} n_{i, k}^{m}}
$$

$D_{c}^{m}$ : diameter of the spiral drum of the shearer $[\mathrm{m}]$.

$a_{\phi}^{m}$ : the number of picks on the $\phi$-th transversal line of the shearer.

The instantaneous total average cutting resistance $Z_{\phi \varphi}^{m}$ of a single pick can be calculated by Formula (2) [15].

$$
Z_{\phi \varphi}^{m}=Z_{0 \phi \varphi}^{m}+f^{m^{\prime}}\left(Y_{\phi \varphi}^{m}-Y_{0 \phi \varphi}^{m}\right)
$$

where:

$Z_{0 \phi \varphi}^{m}$ : instantaneous average cutting resistance of $\varphi$-th conical pick on the $\phi$-th transversal line of the shearer.

$f^{m^{\prime}}$ : coefficient of resistance to cutting coal.

$Y_{\phi \varphi}^{m}-Y_{0 \phi \varphi}^{m}$ : increase in traction force when drum cutting, and $Y_{\phi \varphi}^{m}-Y_{0 \phi \varphi}^{m}=100 \sigma_{y}^{m} s_{d}^{m} k_{\delta}^{m}$.

$\sigma_{y}^{m}$ : unidirectional compressive strength of coal, $\sigma_{y}^{m}=10 f^{m}$, Mpa.

$f^{m}$ : coefficient of coal rock firmness.

$s_{d}^{m}$ : wear area of a conical pick.

$k_{\delta}^{m}:$ volume coefficient of the stress state of coal.

$$
\begin{gathered}
Z_{\phi \varphi}^{m}=Z_{0 \phi \varphi}^{m}+f^{m^{\prime}}\left(Y_{\phi \varphi}^{m}-Y_{0 \phi \varphi}^{m}\right)=Z_{0 \phi \varphi}^{m}+1000 f^{m} f^{m^{\prime}} s_{d}^{m} k_{\delta}^{m} \\
Z_{0 \phi \varphi}^{m}=\frac{10 A^{m}\left(0.35 b^{m}+0.3\right) h_{\phi \varphi, k}^{\bar{m}} l_{\phi}^{m} K_{C}^{m} v_{q i, k}^{m}}{\cos \beta_{\phi}^{m}\left(b^{m}+B^{m} \sqrt{h_{\phi \varphi, k}^{\bar{m}}}\right)}=\frac{120000 A^{m}\left(0.35 b^{m}+0.3\right) l_{\phi}^{m} K_{C}^{m} v_{q i, k}^{m}}{\pi a_{\phi}^{m} n_{i, k}^{m} \cos \beta_{\phi}^{m}\left(b^{m}+B^{m} \sqrt{\frac{12000 v_{q i, k}^{m}}{\pi a_{\phi}^{m} n_{i, k}}}\right)}
\end{gathered}
$$

$A^{m}$ : average cutting resistance of the coal seam.

$b^{m}$ : cutting edge width of a conical pick.

$0.35 b^{m}+0.3$ : reflect the influence of edge cutting width.

$B^{m}$ : brittleness coefficient of coal.

$l_{\phi}^{m}$ : average distance between pick-tip and the center of cutting header [cm].

$K_{C}^{m}=k_{z}^{m} k_{y}^{m} k_{\theta}^{m} k_{c}^{m} k_{t}^{m}$.

$k_{z}^{m}$ : coefficient of the free surface.

$k_{y}^{m}$ : influence coefficient of cutting angle.

$k_{\theta}^{m}$ : influence coefficient of the cutting surface shape of the conical pick.

$k_{c}^{m}$ : coefficient of conical pick arrangement.

$k_{t}^{m}$ : influence coefficient of ground pressure on coal mining face.

$\beta_{\phi}^{m}$ : the deflection angle between the pick axis on the $\phi$-th transversal line relative to the traction direction.

According to the above formulas, the average cutting resistance $f_{j i, k}^{m}(\mathrm{~N})$ of a shearer in the $p r_{i, k}^{m}$ can be calculated by Formula (5).

$$
f_{j i, k}^{m}=0.5 k^{m} a_{i}^{m}\left(\frac{c_{1}^{m} A^{m} v_{q i, k}^{m}}{c_{3}^{m} n_{i, k}^{m}\left(b^{m}+B^{m} \sqrt{\frac{c_{2}^{m} v_{q i, k}^{m}}{n_{i, k}^{m}}}\right)}+c_{4}^{m}\right)
$$

where, 
$k^{m}$ : the number of the transversal lines

$$
\begin{gathered}
c_{1}^{m}=120000\left(0.35 b^{m}+0.3\right) l_{i}^{m} K_{C}^{m} \\
c_{2}^{m}=\frac{12000}{\pi a_{i}^{m}} \\
c_{3}^{m}=\pi a_{i}^{m} \cos \beta_{i}^{m} \\
c_{4}^{m}=1000 f^{m} f^{m^{\prime}} s_{d}^{m} k_{\delta}^{m}
\end{gathered}
$$

It is clear that the cutting resistance of drum in the $p r_{i, k}^{m}$ is a non-linear function of time-variant parameters $A^{m}, v_{q i, k}^{m}, n_{i, k}^{m}$ and $f^{m}$ according to (5).

\subsubsection{Traction Resistance of the Shearer $f_{q i, k}^{m}$}

The traction resistance $f_{q i, k}^{m}(\mathrm{kN})$ of a shearer in the $p r_{i, k}^{m}$ can be estimated by Formula (6) [16].

$$
f_{q i, k}^{m}=\sum_{\lambda=1}^{2} P_{N \lambda}^{m}+f^{\prime}\left(G^{m} \cos \alpha^{m}-\sum_{\lambda=1}^{2} P_{p \lambda}^{m}\right)+f^{\prime \prime} \sum_{\lambda=1}^{2} P_{z \lambda}^{m} \pm G^{m} \sin \alpha^{m}
$$

where:

$\alpha^{m}$ : the inclination angle of coal mining face;

$\pm G^{m} \sin \alpha^{m}$ is the sliding resistance caused by the weight of the shearer, take " + " when pulling upward.

$\sum_{\lambda=1}^{2} P_{N \lambda}^{m}$ is the propulsive resistance of the front and rear drums, which is about $60 \sim 80 \%$ of the cutting resistance of the drums. In this paper, we use 0.8 in the following formula.

$$
\sum_{\lambda=1}^{2} P_{N \lambda}^{m}=\frac{19.1 P_{H}^{m} \eta_{j}^{m}}{D_{c}^{m} n_{i, k}^{m}}\left(1+k_{1}^{m} \frac{h^{m}-D_{c}^{m}}{D_{c}^{m}}\right) \times 0.8
$$

where:

$P_{H}^{m}$ : the rated power of a single cutting motor $[\mathrm{kW}]$.

$\eta_{j}^{m}$ : transmission efficiency of cutting motor.

$h^{m}$ : shearer design height [m].

$k_{1}^{m}$ : working condition coefficient of the rear drum.

$G^{m}$ : shearer weight [t].

The traction resistance $f_{q i, k}^{m}$ of a shearer in the $p r_{i, k}^{m}$ can be calculated as Formula (8).

$$
\begin{gathered}
f_{q i, k}^{m}=0.8 \times \frac{19.1 P_{H}^{m} \eta_{j}^{m}}{D_{c}^{m} n_{i, k}^{m}\left(0.58+0.0067 \alpha^{m}\right)}\left(1+k_{1}^{m} \frac{h^{m}-D_{c}^{m}}{D_{c}^{m}}\right)+\frac{G^{m} \sin \alpha^{m}}{0.58+0.0067 \alpha^{m}}=\frac{c_{5}^{m}}{n_{i, k}^{m}}+c_{6}^{m} \\
\text { where } c_{5}^{m}=0.8 \times \frac{19.1 P_{H}^{m} \eta_{j}^{m}}{D_{c}^{m}\left(0.58+0.0067 \alpha^{m}\right)}\left(1+k_{1}^{m} \frac{h^{m}-D_{c}^{m}}{D_{c}^{m}}\right), c_{6}^{m}=\frac{G^{m} \sin \alpha^{m}}{0.58+0.0067 \alpha^{m}} .
\end{gathered}
$$

\subsection{Power Consumption Model of the Shearer}

3.2.1. Traction Power Consumption of Drum Cutting $p_{j i, k}^{m}$

The cutting power consumption $p_{j i, k}^{m}$ of a shearer in the $p r_{i, k}^{m}$ can be determined according to Formulas (9) and (10).

$$
p_{j i, k}^{m}=\frac{\pi f_{j i, k}^{m} D_{c}^{m} n_{i, k}^{m}}{60000 \eta_{j}^{m}}
$$




$$
p_{j i, k}^{m}=\frac{c_{7}^{m} f_{j i, k}^{m} n_{i, k}^{m}}{\eta_{j}^{m}}=0.5 c_{8}^{m} n_{i, k}^{m}\left(\frac{c_{1}^{m} A^{m} v_{q i, k}^{m}}{c_{3}^{m} n_{i, k}^{m}\left(b^{m}+B^{m} \sqrt{\frac{c_{2}^{m} v_{q i, k}^{m}}{n_{i, k}^{m}}}\right)}+c_{4}^{m}\right)
$$

where $c_{7}^{m}=\frac{\pi D_{c}^{m}}{60000}, c_{8}^{m}=\frac{c_{7}^{m} k^{m} a_{i}^{m}}{\eta_{j}^{m}}$.

\subsubsection{Power Consumption of Shearer Traction $p_{q i, k}^{m}$}

The traction power consumption $p_{q i, k}^{m}(\mathrm{~kW})$ of a shearer in the $p r_{i, k}^{m}$ can be calculated as follows [17]:

$$
p_{q i, k}^{m}=\frac{f_{q i, k}^{m} v_{q i, k}^{m}}{\eta_{q}^{m}}=v_{q i, k}^{m}\left(\frac{c_{5}^{m}}{n_{i, k}^{m} \eta_{q}^{m}}+\frac{c_{6}^{m}}{\eta_{q}^{m}}\right)=v_{q i, k}^{m}\left(\frac{c_{9}^{m}}{n_{i, k}^{m}}+c_{10}^{m}\right)
$$

where $\eta_{q}^{m}$ is the transmission efficiency of the traction motor.

Therefore, the total power consumption $p_{c i, k}^{m}(\mathrm{~kW})$ of a shearer in the $\mathrm{pr}_{i, k}^{m}$ can be expressed as follows:

$$
\begin{gathered}
p_{c i, k}^{m}=\sum_{\lambda=1}^{2} p_{j i, k}^{m}+p_{q i, k}^{m} \\
p_{c i, k}^{m}=c_{8}^{m} n_{i, k}^{m}\left(\frac{c_{1}^{m} A^{m} v_{q i, k}^{m}}{c_{3}^{m} n_{i, k}^{m}\left(b^{m}+B^{m} \sqrt{\frac{c_{2}^{m} v_{q i, k}^{m}}{n_{i, k}^{m}}}\right)}+c_{4}^{m}\right)+v_{q i, k}^{m}\left(\frac{c_{9}^{m}}{n_{i, k}^{m}}+c_{10}^{m}\right)
\end{gathered}
$$

It is clear that the power function of a shearer in the $\mathrm{pr}_{i, k}^{m}$ is a non-linear function of time-variant parameters $v_{q i, k^{\prime}}^{m} n_{i, k^{\prime}}^{m}$ and the coal seam's characteristics parameters $A^{m}, B^{m}$.

\subsubsection{Energy Consumption of the Shearer}

Based on the power consumption derived in Section 3.2.2, we can easily obtain the energy consumption of $p r_{i, k}^{m}$ as Formula (14):

$$
W_{c i, k}^{m}(t)=\int_{t_{i, k}^{s, m}}^{t_{i, k}^{e m}} p_{c i, k}^{m}(t) d t
$$

Thus, the $k$-th production cycle's energy consumption can be calculated as Formula (15):

$$
W_{c, k}^{m}=\sum_{i=1, k \in \mathbf{K}}^{4} W_{c i, k}^{m}(t)=\sum_{i=1, k \in \mathbf{K}}^{4} \int_{t_{i, k}^{s, m}}^{t_{i, k}^{e m}} p_{c i, k}^{m}(t) d t
$$

The total energy consumption of the $m$-th coal mining face is as Formula (16).

$$
W_{c}^{m}=\sum_{k=1}^{K} \sum_{i=1}^{4} W_{c i, k}^{m}(t)=\sum_{k=1}^{K} \sum_{i=1}^{4} \int_{t_{i, k}^{s, m}}^{t_{i, k}^{m}} p_{c i, k}^{m}(t) d t
$$

$\mathbf{K}$ : set of the production cycle at the $m$-th coal mining face, $\mathbf{K}=\{1,2, \ldots k, k+1, \ldots K\}$.

\section{Optimization of Energy Consumption Considering a Mining Cycle}

\subsection{Objective Function}

The objective of the optimization is to minimize the energy consumption of the whole coal mining process, which can be expressed as (17).

$$
\min W=\sum_{m=1}^{M} W_{c}^{m}
$$


$\mathbf{M}$ : the set of coal mining face, $\mathbf{M}=\{1,2, \ldots m, m+1, \ldots M\}$.

\subsection{Constraints}

4.2.1. Range of Traction Speed $v_{q i, k}^{m}$, Drum Cutting Rotational Speed $n_{i, k}^{m}$ of Shearer in k-th Production Cycle

$$
v_{q i, k}^{m, \min } \leq v_{q i, k}^{m}(t) \leq v_{q i, k}^{m, \max }
$$

where $v_{q i, k}^{m, m i n}$ and $v_{q i, k}^{m, m i n}$ represent the minimum and maximum traction speed limits of the shearer for $\mathrm{pr}_{i, k}^{m}$ respectively.

$$
n_{i, k}^{m, \min } \leq n_{i, k}^{m}(t) \leq n_{i, k}^{m, \max }
$$

where $n_{i, k}^{m, \min }$ and $n_{i, k}^{m, \min }$ represent the minimum and maximum drum cutting rotational speed limits of the shearer for $\mathrm{pr}_{i, k}^{m}$ respectively.

The constraints (18)-(19) are set for the operational limits of the shearer.

\subsubsection{Mining Time}

(1) Total mining time

$$
\sum_{i=1, k \in K}^{6} t_{p i, k} \leq T_{k}^{\max }
$$

where $T_{k}^{\max }$ represent the maximum allowable coal mining time in $k$-th production cycle.

$$
\sum_{k=1}^{K} \sum_{i=1}^{6} t_{p i, k} \leq T_{c}^{\max }
$$

where $T_{c}^{\max }$ represent the maximum allowable coal mining time for the whole production of $m$-th mining face. The constraints (18)-(19) are set for the maximum allowable mining time.

(2) Working time of coal mining

$$
\left\{\begin{array}{c}
T_{c, k}^{m}=t_{c, k}^{e, m}-t_{c, k}^{s, m} \leq T_{c, k}^{m, \text { goal }} \\
T_{c}^{m}=\sum_{k=1}^{K}\left(t_{c, k}^{e, m}-t_{c, k}^{s, m}\right) \leq T_{c}^{m, \text { goal }}
\end{array}\right.
$$

where $T_{c, k}^{m}$ represent the working time of coal mining in $k$-th production cycle, and $T_{c, k}^{m, g o a l}$ represent the requirement of coal mining time in $k$-th production cycle. $T_{c}^{m}$ represent the working time of coal mining for $K$ production cycles, and $T_{c}^{m, g o a l}$ represent the requirement of coal mining time for $K$ production cycles. The constraints (22) are set for the requirement of mining time.

\subsubsection{Production Requirement}

$$
\left\{\begin{array}{c}
Q_{c, k}^{m}=10^{3} h^{m} s^{m} \gamma_{k}^{m} c^{m}\left(\sum_{i=1}^{6} \mu_{i}^{m} \int_{t_{i, k}^{s, k}}^{t_{i, m}^{e, m}} v_{q i, k}^{m}(t) d t\right) \geq Q_{c, k}^{\min } \\
Q_{c}^{m}=10^{3} h^{m} s^{m} \gamma_{k}^{m} c^{m}\left(\sum_{k=1}^{K} \sum_{i=1}^{6} \mu_{i}^{m} \int_{t_{i, k}^{t, m}}^{t_{i, k}^{e, m}} v_{q i, k}^{m}(t) d t\right) \geq Q_{c}^{\min }
\end{array}\right.
$$

where:

$\mu_{i}^{m}:$ coal cutting coefficient in $p r_{i, k^{m}}^{m}$

$\mu_{1}^{m}+\mu_{2}^{m}+\mu_{31}^{m}=1 ; \mu_{32}^{m}=1 ; \mu_{4}^{m} \stackrel{i,{ }^{\prime}}{=}$;

where $Q_{c}^{m}$ represents the amount of coal mined by the shearer during the whole mechanized mining period. 
The constraints (23) are set for the requirement of mining output.

\section{Simulation, Results and Discussion}

In this paper, the 91xx mining face of Wang Zhuang coal mine in Shanxi province, China, using the MG400/940-WD shearer is employed as a study case to illustrate the validity of the proposed models, and the effects of the optimal control on the shearer's energy consumption. Based on the energy consumption model of shearer that established in Section 3, the relationships among energy consumption of shearer, coal mining output, traction speed, drum rotational speed and effective mining time are analyzed, then control and coordination strategies between the traction speed, drum rotational speed are proposed to minimize the shearer's energy consumption.

The simulation is programmed in Matlab 2017b installed on a ThinkPad personal computer running windows-10 professional 64 bit with Intel(R) Core(TM) i5-8250U central processing unit (CPU), 8.00 GB RAM. The non-linear constraint optimization function "fmincon" using the interior-point method in Matlab is used to solve the optimization problem.

It should be specified that all the relevant speed $v_{q i, k}^{m}$ and $n_{i, k}^{m}$ in $\operatorname{pr}_{i, k}^{m}$ can be controlled and measured from the operation system. The traction speed and drum rotational speed are usually a constant value in each $p r_{i, k}^{m}$, therefore, in this paper, we assume that the aforementioned speeds do not change during the entire $p r_{i, k}^{m}$. In addition, to simply propose the formulation of the related energy consumption of the entire production process, we redefine the speed of each process as follows:

$$
\begin{aligned}
& v_{q i, k}^{m}(\mathbf{t})=\left\{\begin{array}{rr}
v_{q i, k^{\prime}}^{c, m} & t_{i, k}^{s, m} \leq t \leq t_{i, k}^{e, m} \\
0, & \text { otherwise }
\end{array}\right. \\
& n_{i, k}^{m}(\mathbf{t})=\left\{\begin{array}{cc}
n_{i, k}^{c, m}, & t_{i, k}^{s, m} \leq t \leq t_{i, k}^{e, m} \\
0, & \text { otherwise }
\end{array}\right.
\end{aligned}
$$

\subsection{Simulation Parameters}

Tables 1 and 2 depict the related parameters of the 91xx mining face and MG400/940WD shearer used in Section 3.

Table 1. Parameters of the $91 x x$ mining face.

\begin{tabular}{ccc}
\hline$L^{m}: 260 \mathrm{~m}$ & $x_{p}^{m}: 45 \mathrm{~m}$ & $\alpha^{m}: 6$ \\
\hline$s^{m}: 0.8 \mathrm{~m}$ & $h^{m}: 3.3 \mathrm{~m}$ & $\gamma_{k}^{m}: 1.42 \mathrm{t} / \mathrm{m}^{3}$ \\
\hline$c^{m}: 0.98$ & & \\
\hline
\end{tabular}

Table 2. Parameters of MG400/940-WD shearer.

\begin{tabular}{ccc}
\hline$d_{s}^{m}: 10 \mathrm{~m}$ & $G^{m}: 60 \mathrm{~m}$ & $P_{H}^{m}: 400 \mathrm{~kW}$ \\
\hline$D_{c}^{m}: 2 \mathrm{~m}$ & $\eta_{j}^{m}: 0.8$ & $\eta_{q}^{m}: 0.8$ \\
\hline$k_{1}^{m}: 0.8$ & & \\
\hline
\end{tabular}

\subsection{Analysis of Simulation Result}

This paper mainly analyzes the changes in energy consumption of a shearer with the traction speed, drum rotational speed and effective mining time when the shearer conducts oblique cutting and linear coal cutting. It should be noted that the influences on energy consumption of what we have studied are all under ideal working conditions, such as invariable average cutting resistance of coal seam in the longwall face. 


\subsubsection{Energy Consumption and Mining Output with Several Speed Coordination Cases}

Many factors such as the magnitude and the rate of change of $v_{q i, k}^{c, m}$ and $n_{i, k}^{c, m}$ can directly affect the energy consumption of the shearer. In Section 5.2.1, several speed coordination cases are analyzed to gain an insight into the impact of traction speed and drum rotational speed on the shearer's energy consumption.

Based on the actual operation of the shearer in unidirectional mining of the 91xx mining face, the base value of $v_{q i, k}^{c, m}$ and $n_{i, k}^{c, m}$ when $i=4$ are shown in Table 3. According to this base value, case $1 \sim$ case 4 with various speed combinations [traction speed (tra-speed) and drum speed (dr-speed)] in the reasonable range are designed in Figure 2. Case 5 shown in Table 4 is designed for analyzing the shearer's energy consumption distribution conveniently. It should be noted that the speeds (p.u.) (Hereinafter, all the speed in p.u. are calculated with the base speed in Table 3, e.g., a traction speed of $0.03(\mathrm{~m} / \mathrm{s})$ in process 1 is $0.03 / 0.025=1.2$ (p.u.), where 0.025 is the base traction speed of process 1 (Table 3 ).) in the five cases are constant value in each process of a production cycle, Figures $3-5$ show the relevant results of selected combinations of varies $v_{q i, k}^{c, m}$ and $n_{i, k}^{c, m}$.

Table 3. The base value of speed.

\begin{tabular}{ccccc}
\hline Process & Pr1 & Pr2 & Pr3 & Pr4 \\
\hline traction speed $(\mathrm{m} / \mathrm{s})$ & 0.025 & 0.0333 & 0.0367 & 0.0467 \\
drum speed $(\mathrm{r} / \mathrm{min})$ & 25 & 25 & 30 & 25 \\
\hline
\end{tabular}
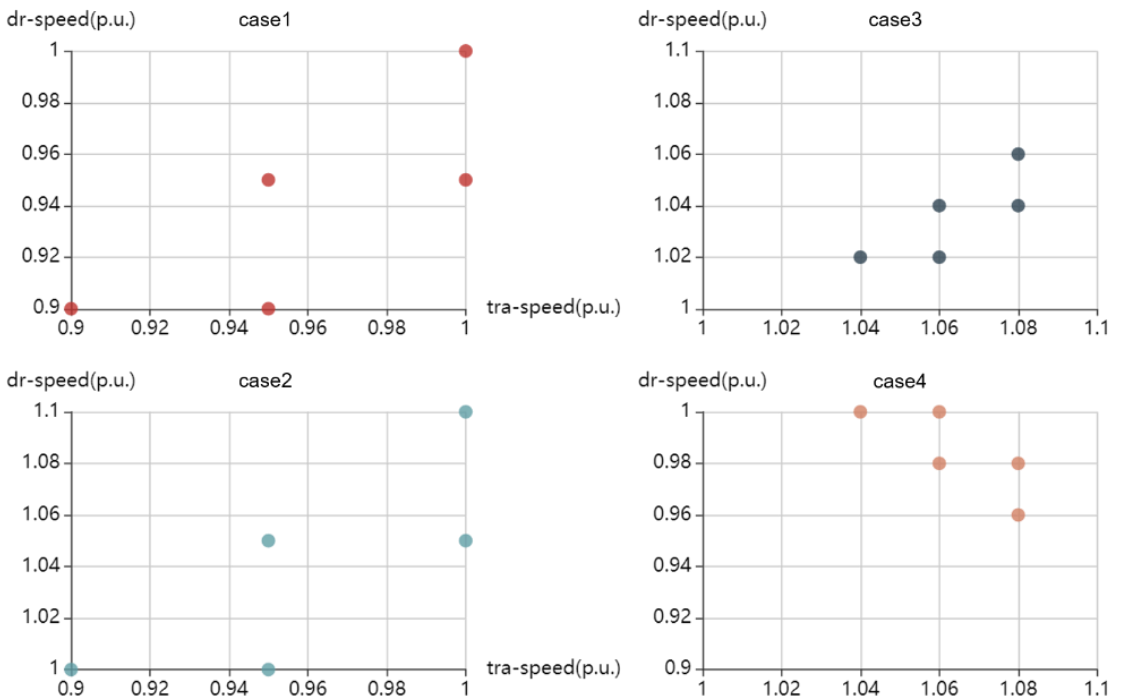

Figure 2. Designed cases with the speed combinations in a reasonable range.

Table 4. Parameters of case 5.

\begin{tabular}{ccccccc}
\hline Case & Speed & \multicolumn{5}{c}{ Speed Value [p.u.] } \\
\hline Case 5 & traction speed & 0.9 & 0.9 & 0.95 & 1 & 1.05 \\
& Drum speed & 0.95 & 1.05 & 1.1 & 0.9 & 0.95 \\
\hline
\end{tabular}

The mathematical model of the shearer proposed in Section 3 is simulated according to case $1 \sim$ case 4 in Figure 2 and case 5 in Table 4, and the simulation results of the energy consumption of the shearer can be obtained in Figure 4 (e.g., case 1). The $x$-axis of Figure 4 represents the mining time, the $y$-axis denotes the energy consumption of the shearer in a production cycle. It is manifest that with the same drum speed, the energy consumption is decreased with the increasing of the traction speed, however, it is decreased with the decreasing of the drum speed under the condition of the same traction speed. 
Figure 4 shows the mining output with varied $v_{q i, k}^{c, m}$ and $n_{i, k}^{c, m}$ of case 1 in a production cycle, and the coal production of the mining face in a production cycle is about 955,194 (kg).

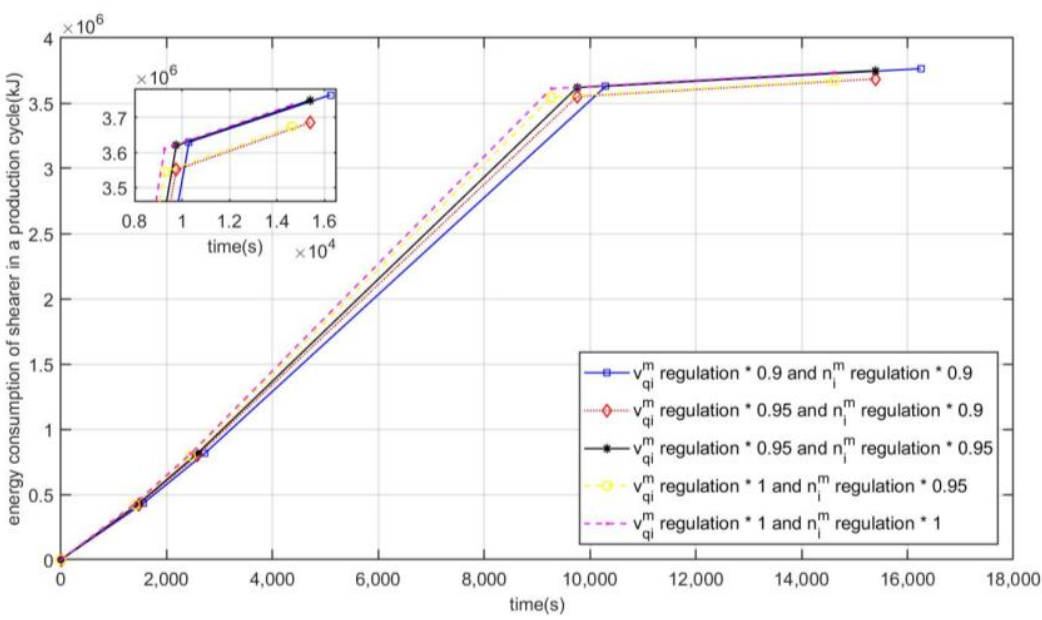

Figure 3. The shearer's energy consumption of case 1 with varied $v_{q i, k}^{c, m}$ and $n_{i, k}^{c, m}$ in a production cycle.

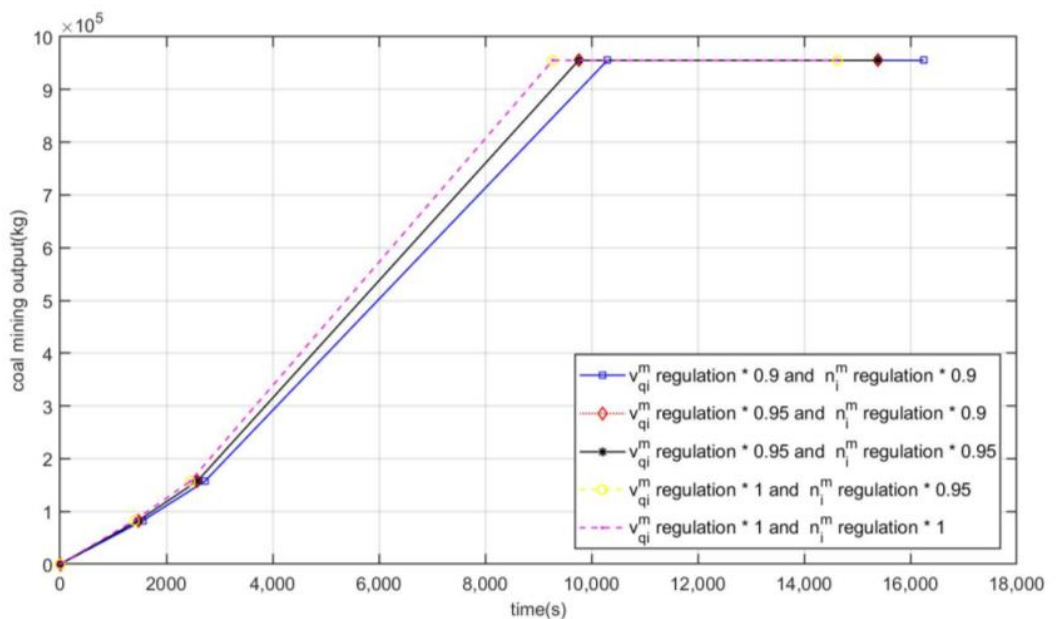

Figure 4. The coal mining output with varied $v_{q i, k}^{c, m}$ and $n_{i, k}^{c, m}$ of case 1 in a production cycle.

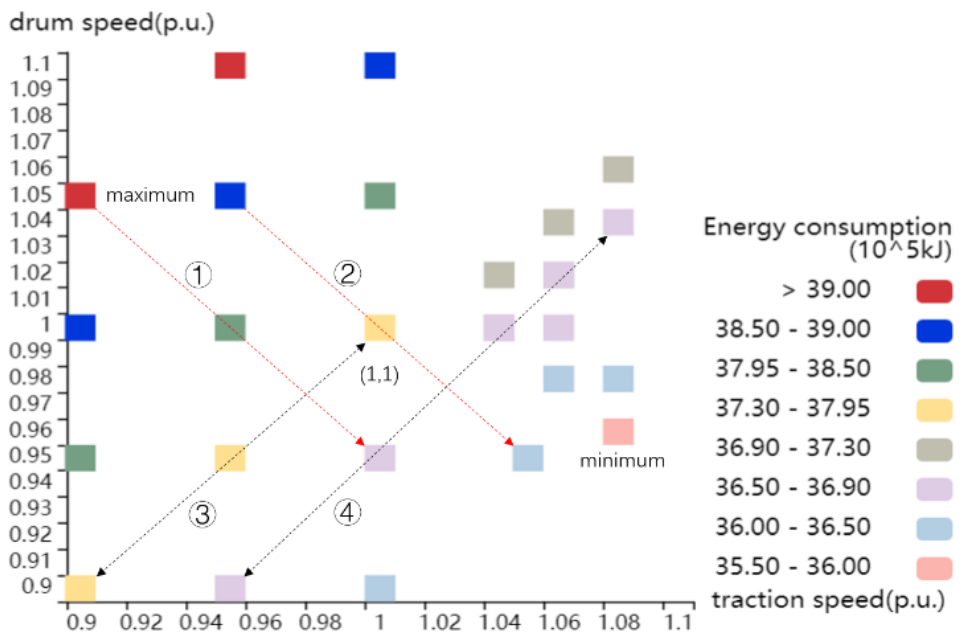

Figure 5. The energy consumption of shearer under 5 cases in a production cycle. 
A comparison and analysis of the energy consumption of a shearer based on the speed combination (SC: traction speed and drum speed) which is given in Figure 2 and a column in Table 4 are simulated and presented in this paper. The $x$-axis of Figure 5 (energy consumption thermodynamic diagram) represents the traction speed (p.u.), the $y$-axis denotes the drum speed (p.u.), and different colors are used to distinguish the value of energy consumption.

The results of Figure 5 are as follows,

(1) The maximum energy consumption of the shearer is 3,964,775 $\mathrm{kJ}[\mathrm{SC}=(0.9,1.05)$ (SC = [traction speed (p.u.), drum speed (p.u.)]) in case 5], and the minimum energy consumption of the shearer is $3,578,145 \mathrm{~kJ}$ [SC $=(1.08,0.96)$ in case4]. With the same production constraints, the energy consumption can be saved $9.75 \%$ by coordinating the traction speed and drum speed.

(2) Combinations of case 1 and case 2 which with consistent speed variation are selected to analyze the influence of changing traction speed and drum speed on the shearer's energy consumption.

The energy consumption of the shearer is $3,749,344 \mathrm{~kJ}[\mathrm{SC}=(0.95,0.95)$ in case 1], while the drum speed remains unchanged at 0.95 , the energy consumption of shearer decreased by $2 \%$ with the traction speed increasing 0.05 , while the traction speed remains unchanged at 0.95 , the energy consumption of shearer decreased by $1.7 \%$ with the drum speed decreasing 0.05 .

The energy consumption of the shearer is $3,877,114 \mathrm{~kJ}$ [SC $=(0.95,1.05)$ in case2], while drum speed remains unchanged at 1.05, the energy consumption of the shearer decreased by $2.07 \%$ with the traction speed increasing 0.05 , while traction speed remains unchanged at 0.95 , the energy consumption of the shearer decreased by $1.65 \%$ with the drum speed decreases 0.05 .

In conclusion, compared with the decrease of drum speed, the increase of traction speed has a greater impact on the energy consumption of the shearer, and the result will be consistent with other speed combinations which have the same speed variation.

To guide the actual coal mining, the paths (1) (2) in Figure 5 are selected to analyze the most obvious energy-saving ways on how to coordinating the traction speed and drum speed. The energy consumption of the minimum is reduced by $7.32 \%$ and $7.01 \%$ compared with the maximum value along the paths (1) and (2), respectively. Other paths with the same variation rule of speeds as path (1) (2) can be analyzed similarly.

The paths (3) (4) in Figure 5 are selected to analyze the distribution characteristics of energy consumption from another perspective. Along the paths (3) or (4), there is no significant difference in the energy consumption of the shearer which has the same color; it changed slightly when the traction speed and drum speed increased or decreased synchronously. Other paths with the same variation rule of speeds as path (3) (4) can be analyzed similarly.

In conclusion, considering the conditions of coal production, effective mining time and the structural parameters of the shearer, the energy consumption of the shearer can be reduced by coordinating the traction speed and drum speed effectively.

\subsubsection{Energy Consumption and Mining Output with Optimal $v_{q i, k}^{c, m}$ and $n_{i, k}^{c, m}$ and Other} Analyzed Speed Coordination Cases

In this section, we minimized the total energy consumption of the shearer in a production cycle with optimal $v_{q i, k}^{c, m}$ and $n_{i, k}^{c, m}$ in each $\operatorname{pr}_{i, k}^{m}$. The optimized speeds are shown in Table 5 (take the corresponding base value in Table 3 as the reference value). It should be noted that the traction speed and drum speed remain constant in each optimization process in a production cycle.

Compared with the energy consumption values described in Figures 3 and 6, we can see that the optimized total energy consumption reduced by $5.98 \%, 3.87 \%, 5.51 \%, 3.58 \%$ and $5.16 \%$, respectively, and the optimal total energy consumption in a production cycle 
was 3,542,908 kJ. Furthermore, Figure 7 depicts the coal mining output with optimal $v_{q i, k^{\prime}}^{m}$ $n_{i, k}^{m}$ of different processes and other analyzed speed combinations of case 1, compared with the mining output described in Figures 4 and 7; we can see that the total coal production is $955,194(\mathrm{~kg})$ as in the other five speed combinations. Other cases can be analyzed similarly and the results were constant with case 1 .

Table 5. Optimized value of speed [p.u.].

\begin{tabular}{ccccc}
\hline Process & Pr1 & Pr2 & Pr3 & Pr4 \\
\hline traction speed & 1.168 & 1.0511 & 1.0654 & 1.1156 \\
drum speed & 0.92 & 0.92 & 0.9333 & 0.8 \\
\hline
\end{tabular}

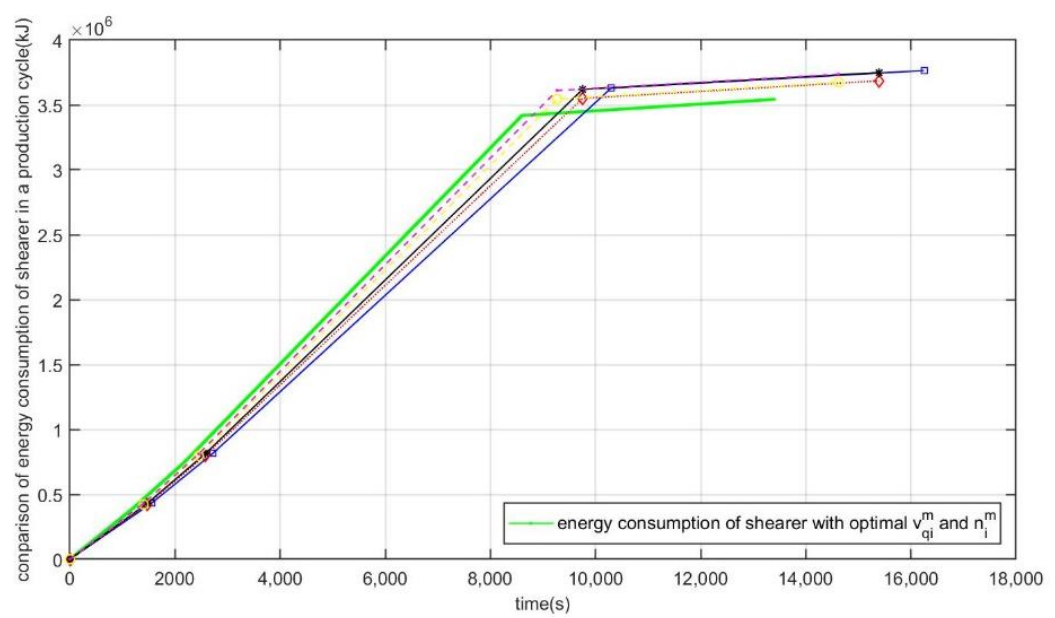

Figure 6. The energy consumption of shearer with optimal and other analyzed speed combination of case 1.

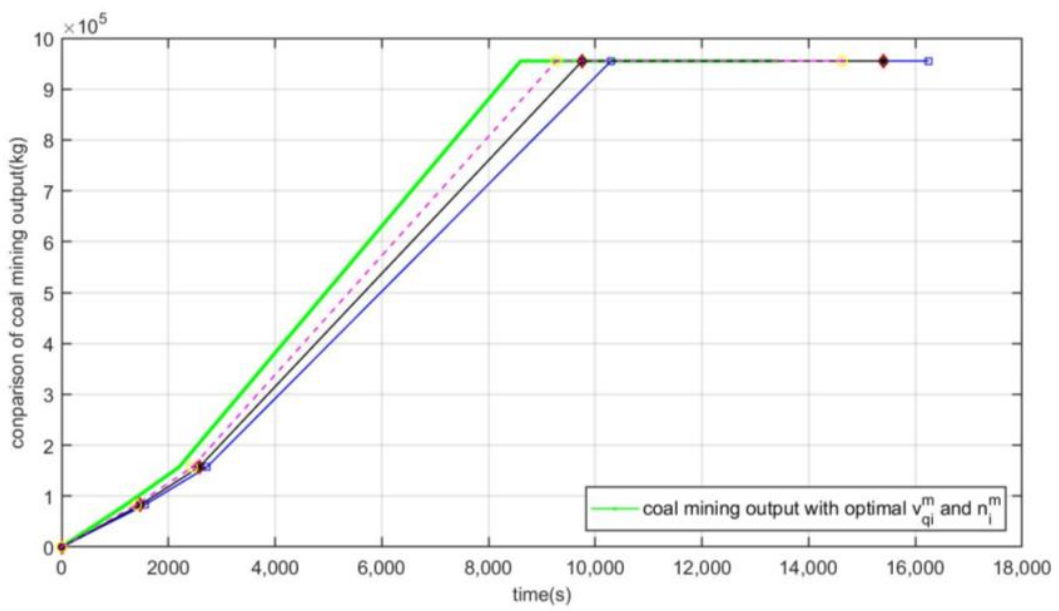

Figure 7. The coal mining output with optimal and other analyzed speed combination of case 1.

The radius-axis of Figure 8 (columnar stack diagram in polar coordinates) represents the energy consumption of the shearer in each process in a production cycle, the angle-axis denotes the speed combinations of five cases and the optimized, and different colors are used to distinguish the energy consumption of each process. According to Figure 8, we can see that after the optimization, the energy consumption of the shearer reduced effectively. Compared with the base value, the energy consumption was reduced by $5.16 \%$, and $10.64 \%$ compared with the largest combination ( $\mathrm{SC}=0.9,1.05)$, and $0.98 \%$ in comparison with the lowest combination $(\mathrm{SC}=1.08,0.96)$. 
If we consider other benefits brought by the coordination control of speeds, such as requirements for ventilation, the reduction of dust, etc., the optimization of the mining would have more real implications.

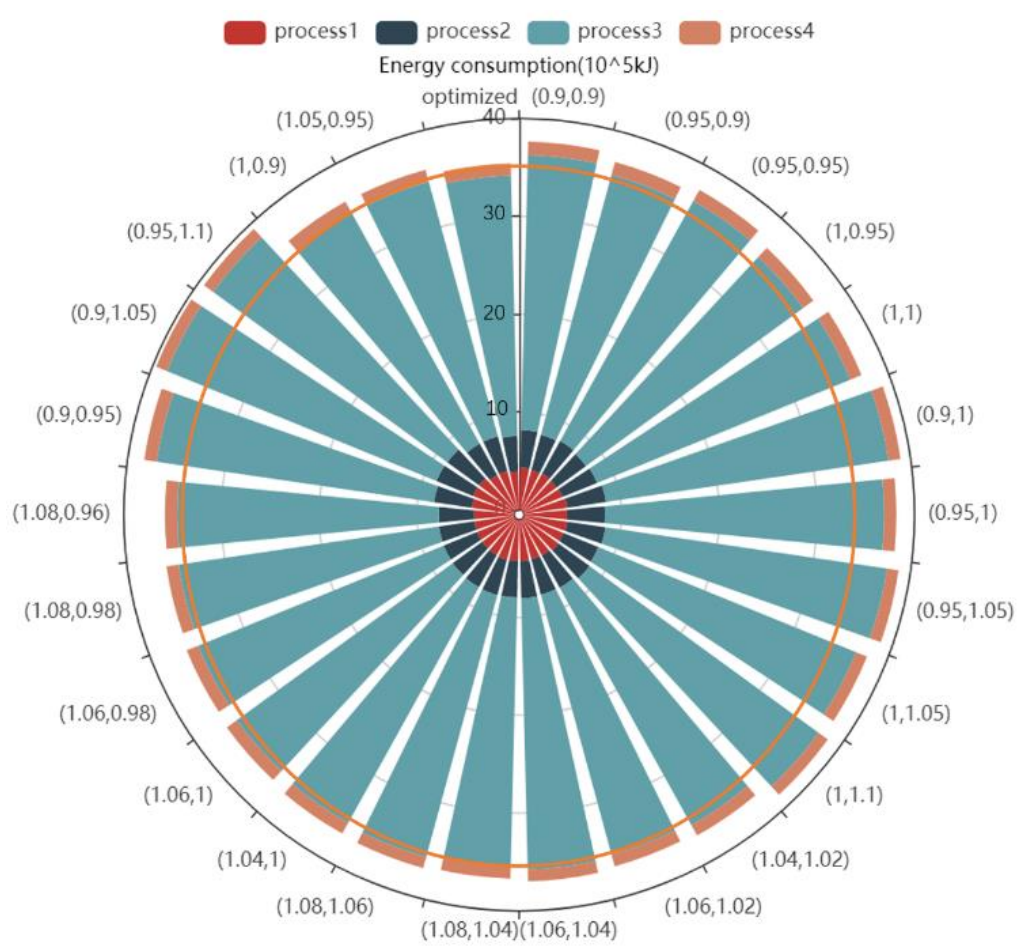

Figure 8. The energy consumption of shearer with optimal and other analyzed speeds rate combination of 5 cases.

\section{Conclusions}

The drum shearer, accounting for the second-largest proportion of energy consumption of the equipment used in a mining subsystem, is one of the critical factors deciding the efficiency and energy consumption of coal mining. In this paper, the 91xx mining face of Wang Zhuang coal mine in Shanxi province, using an electric haulage drum shearer, was employed as a study case. Based on the analysis of the cutting and traction resistances on the shearer during different processes, an energy consumption model of a shearer including a function of the traction speed, the drum rotational speed and the effective coal mining time, was established, which was suitable for the optimal control of the shearer. Moreover, to give practical implications for how to achieve greater energy-saving production, several speed coordination cases were employed to further analyze the impact of the traction speed and the drum rotational speed on the energy consumption of the shearer. Aiming at minimizing the total energy consumption of the shearer in a production cycle, optimal control and coordination strategies between the traction speed and drum rotational speed were proposed. It has to be highlighted that optimal control and coordination strategies of a shearer can effectively reduce the energy consumption of $5.16 \%$ in a production cycle while considering all the constraints such as the same coal-mining output, and a certain period of time.

Our study can give the best and practical guidance of the speed coordination as a means to possibly approach more energy-saving production during complex coal mining, and it can help to achieve the lean management of a coal mine.

In future studies, possible improvements may include a comprehensive analysis of the cooperative operation system of a double-drum shearer and scraper conveyor, and consider the disadvantages of working conditions, such as complicated and constantly changing cutting resistance of a coal seam in a fully mechanized coal mining face. 
Author Contributions: The paper is the cooperative effort of all the authors. Conceptualization, Z.Z. and T.H., methodology, D.C. and T.H.; software, D.C.; validation, Z.Z. and T.H.; formal analysis, D.C. and T.H.; investigation, D.C. and G.Z.; resources, G.Z.; data curation, D.C.; writing-original draft preparation, D.C. and T.H.; writing-review and editing, D.C. and T.H.; visualization, D.C. and T.H.; supervision, Z.Z. and T.H.; project administration, Z.Z. and T.H.; funding acquisition, Z.Z. All authors have read and agreed to the published version of the manuscript.

Funding: This research received no external funding.

Informed Consent Statement: Not applicable.

Data Availability Statement: Data sharing not applicable.

Acknowledgments: This research is supported by The Science and Technology Planning Project of Henan Province of China (No.192102210228) and Henan Mine Power Electronics Device and Con-trol Innovative Technology Team (No. CXTD2017085).

Conflicts of Interest: The authors declare no conflict of interest.

\section{References}

1. China National Energy Administration. The 13th Five-Year Plan for Coal Industry Development; China National Energy Administration: Beijing, China, 2016.

2. Li, J.Z.; Gao, Z.X. Coordinative control of large-scale electro-mechanical equipment in mine. Ind. Mine Autom. 2017, 43, 15-19.

3. Snopkowski, R.; Napieraj, A.; Sukiennik, M. Method of the assessment of the influence of longwall effective working time onto obtained mining output. Arch. Min. Sci. 2017, 61, 967-977. [CrossRef]

4. Okolnishnikov, V. An MTSS Based Underground Coal Mining Simulation Model Engineering. Technol. Appl. Sci. Res. 2018, 8, 3060-3063.

5. Ordin, A.A.; Metelkov, A.A. Optimization of the Fully-Mechanized Stoping Face Length and Efficiency in a Coal Mine. J. Min. Sci. 2013, 49, 254-264. [CrossRef]

6. Bołoz, Ł. Longwall shearers for exploiting thin coal seams as well as thin and highly inclined coal seams. MIAG 2018, 2, 59-72. [CrossRef]

7. Qin, D.T.; Wang, Z.; Hu, M.H.; Liu, Y.G.; Ge, S.S. Dynamic matching of optimal drum movement parameters of shearer based on multi-objective optimization. J. China Coal Soc. 2015, 40, 532-538.

8. Krauze, K.; Mucha, K.; Wydro, T. Evaluation of the Quality of Conical Picks and the Possibility of Predicting the Costs of Their Use. MAPE 2020, 3, 491-504. [CrossRef]

9. Zhao, L.J.; Xi, N.; Wang, Y.; Cui, X.D.; Shi, L. Study on multi-parameter optimization of the shearer's spiral drum in complex coal seams. J. Mach. Des. 2019, 36, 65-71.

10. Tao, C.D. Calculation and application of energy consumption characteristics of drum shearer. J. China Univ. Min. Technol. 1990, 19.

11. Bołoz, Ł.; Biały, W. Automation and Robotization of Underground Mining in Poland. Appl. Sci. 2020, 10, 7221. [CrossRef]

12. Hao, Z.Y.; Zhou, Z.Q.; Zhang, P.; Yang, Q.C. Experimental study on cutting energy consumption of coal mining under different working conditions. J. Mech. Strength 2019, 41, 850-857.

13. Chen, D.; Zheng, Z.; Huang, T.; Zhang, G. A Study on the Optimized Working Schedule of the Fully Mechanized Coal Mining. In Proceedings of the 55th International Universities Power Engineering Conference (UPEC), Turin, Italy, 1-4 September 2020; pp. 1-6.

14. Evans, I. Theory of the cutting force for point-attack picks. Int. J. Rock Mech. Min. Sci. Geomech. Abstr. 1984, 2, 63-71. [CrossRef]

15. Tao, C.D.; Yang, C.M.; Li, B. Mining Machinery; Coal Industry Publishing House: Beijing, China, 1993.

16. Liu, C.S.; Chen, X.P. Theoretical method of matching between traction power and cutting power of shearer. J. Heilongjiang Statute Sci. Technol. 2011, 21, 57-60.

17. Zhou, X. Research on the Key Technologies of Cooperative Control for Equipments in the Fully Mechanized Coal Face; China University of Mining and Technology: Beijing, China, 2014. 